\title{
The Development and Measurement of Identity across the Physical Sciences
}

\author{
Geoff Potvin and Zahra Hazari
}

Department of Engineering \& Science Education, Clemson University

\begin{abstract}
Drawing from earlier work of Gee, Carlone, Johnson, and Shanahan, we developed a framework for "good physics student role identity" or, more simply, "physics identity" which is a reliable proxy for students' affinity towards physics and is predictive of students' physics-related career choices. This framework was postulated to be comprised of performance beliefs, competence beliefs, recognition beliefs, and interest. Subsequent investigations showed that performance and competence beliefs are not distinct and the combined performance/competence construct is somewhat akin to Bandura's self-efficacy. Recent work has extended this framework to mathematics and engineering. We conclude with a brief discussion of the future of the framework for understanding "best practices" in STEM classrooms.
\end{abstract}

Keywords: physics identity, career choice

PACS: $01.30 . \mathrm{Cc}, 01.40 . \mathrm{Fk}$

\section{INTRODUCTION}

The goal of this paper is to recount the development and related measurements of physics identity as a framework, as it has progressed in our research over some years. The intention is to recount in a coherent way the ways in which our framework has changed and improved based on various projects and sources of data; some of which has been published or presented elsewhere and some of which has not. We begin with a brief review of our original conception of identity in relation to prior work in this area, and then discuss results from the projects that have driven the development of our framework and ongoing research questions.

\section{BACKGROUND}

In conceptualizing science identity and, more specifically, physics identity we originally drew insight from the foundational landscape for identity research in education laid out by Gee $[1,2]$ which defined identity in terms of being recognized as a certain "kind of person". This led to a central follow-up question: recognized by whom? In framing our approach to identity and the associated methodological choices we made, it was important to understand how to operationalize this conceptualization of identity. Some identity frameworks are based largely on individual's social performances or external manifestations (what they do, how they act out who they are) that lead to others perceiving them as a certain "kind of person" while other identity frameworks focus on internal states in terms of how students see themselves as a certain "kind of person" [3]. In our work, we found a purely external conceptualization of identity to be limited since it disregards internal states that reflect personal thoughts and emotions which are fundamental to other internal processes such as learning. In other words, what a person does and how a person acts as perceived by others is an incomplete picture of how s/he perceives her/himself and what that means for her/his educational choices. If a person is recognized by others as a certain "kind of person" but does not recognize her/himself as such and is unaware of being identified as such, then the external conceptualization of identity by others (including researchers) may provide limited insight into internal states which strongly influence educational outcomes for that individual (e.g. course persistence/performance, conceptual understanding, procedural knowledge, career decisions). Thus, our work, along with the work of other researchers have expanded the focus of identity research to notions of self-recognition that move beyond purely external manifestations to include internal conceptualizations of who a person perceives her/himself to be $[4,5,6]$.

It is important to note that, similar to Carlone and Johnson [5, 7], our methodological approach frames physics identity as reflexive, where perceptions are aggregated through conscious reflection rather than in-themoment perceptions that are non-reflexive. LichtwarckAschoff [8] delineate these two approaches as being distinct in time scale, the former being macro-level scales used to study long-term changes in identity while the latter are micro-level scales used to study changes closer to real-time. In addition, we conceptualize identity as a "quasi-trait"[9]; that is, it is a reasonably stable trait which can change over time, given certain experiences or circumstances (e.g. an individual's physics identity may 
improve given good experiences in a physics classroom).

\section{PHYSICS IDENTITY FRAMEWORK}

Our framework draws from Carlone and Johnson [5] who documented the importance of three factors to science identity, namely, perceived recognition, belief in the ability to perform, and belief in one's competence. This framework was developed through qualitative research with female scientists in which the participants reflected on their experiences in science and articulated how those experiences impacted how they saw themselves. Our initial framework (described in detail below) deviated from Carlone and Johnson's framework in three ways. First, because the population in which we were interested is students, not scientists, we added the factor of interest. For scientists, interest in science is already established and thus in Carlone and Johnson's study there was likely very little variability across the scientists in terms of their general science interest - not enough to explain differences in science identity. For students, however, the development of interest is central to the development of identity, which was acknowledged in the work of Carlone and Johnson. Our second deviation from this work was our conceptualization of self-recognition (recognizing oneself as a certain "kind of person") as a core feature of having a certain identity whereas Carlone and Johnson incorporated this self-recognition as a part of the recognition factor which included recognition by others. Thus, in our work, seeing oneself as a "physics person" was a core feature of physics identity which was influenced by whether the individual felt recognized by others as a "physics person", was interested in physics, felt able to perform in physics, and felt competent to understand physics. Finally, while Carlone and Johnson use a qualitative approach, which allows for depth in studying identity reflexively, we employ a quantitative approach which allows for a wider sweep of the population but less depth in our identity measures.

Our initial framework for physics identity is laid out in Figure 1. In this framing, a person possesses multiple identities that interact and intersect. Some of these identities are driven by individual factors (personal identities) while others are driven by association with groups (social identities). For students, physics identity is most often a social identity derived from associations and recognition within a physics-related community of practice (e.g. physics class, club, etc.) but it can also be a personal identity. Furthermore, students' physics identities interact with other ways in which they see themselves both individually (e.g. as nurturing, competitive, etc) or as members of a group (e.g. being female, an athlete, etc). Looking more specifically at the development of physics identity, we built on the aforementioned research to propose that it is influenced by students' perceptions of how they are recognized (or not) by others with respect to physics (recognition factor), their interest in physics (interest factor), their beliefs in their ability to perform physics-related tasks (performance factor), and their beliefs in their ability to comprehend physics content (competence factor). All of these perceptions are contingent on students' perceptions of what physics is, which may change after taking a physics class. This framework was used in Hazari et al [10] to guide the quantitative development of a proxy for physics identity which was found to be a significant predictor of the likelihood of choosing a career in physics.

\section{THE RELATIONSHIP BETWEEN PERFORMANCE AND COMPETENCE BELIEFS}

To address what we perceived as a technical limitation of Hazari et al [10] - namely, the inability to statistically distinguish between performance and competence beliefs in our physics identity model - we subsequently designed and tested an improved instrument to assess physics identity and to establish reliable items that would disaggregate performance and competence. Beginning in the summer of 2011, we collated the items from our previously used surveys and designed several new items focusing primarily on performance and competence beliefs, both in the specific domain of physics and in science generally. In the fall semester, we recruited student participants to complete the new instrument on two separate occasions for reliability and validity testing. In total, we recruited participants from two classes totaling about 275 individuals at each of the two data collection points, receiving an approximately $90 \%$ response rate each time.[11, 12]

Performing an exploratory factor analysis on this data using $\mathbf{R}$, the statistical programming language and software environment [13] showed a somewhat unexpected result. Rather than disaggregating performance and competence beliefs, we found that the data showed students to have mixed performance/competence beliefs that depended on the academic/social context. Specifically, we found that students' performance/competence beliefs fell into one of three contextual categories: academic/classroom, interpersonal/conversational, and laboratory/experimental contexts. These factors, along with the expected factors of physics interest, science interest, physics recognition beliefs, and science recognition beliefs, accounted for $68 \%$ of the total variance in the surviving 28 items (all Likert-type scales) in our revised instrument. There was a high degree of internal consistency, with a measured Cronbach's $\alpha$ of 0.95, and 


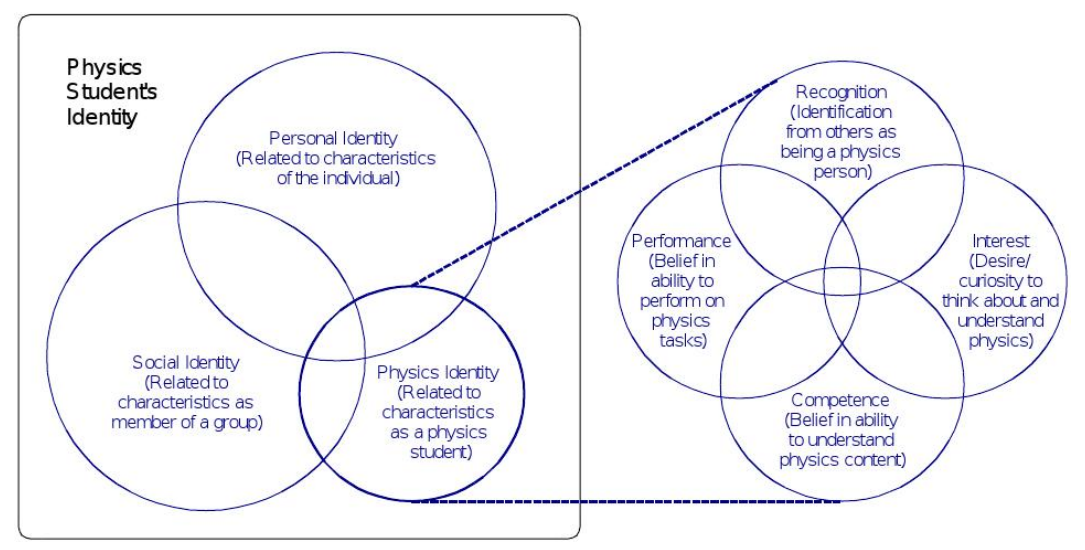

FIGURE 1. Four-factor physics identity model, as described in Hazari et al [10].

the test-retest reliability was found to be good-to-high, with a mean pre-post correlation of 0.695 (ranging from $0.513-0.823$ ). Two subsequent rounds of data collection have confirmed the performance/competence findings, with similar reliabilities.

These findings prompted us to modify our perspective on our model of physics identity, to a threefactor proxy including recognition, interest, and performance/competence beliefs. There is some similarity between our revised performance/competence factor and the self-efficacy construct originally developed by Bandura [14], which is a proxy for one's belief in one's own ability to complete tasks in a particular domain.

\section{MOVING BEYOND THE CONTEXT OF PHYSICS}

With these new perspectives on our model of physics identity, we set out to expand the study of these results through more comprehensive and sophisticated analytic methods, as well as expand the discussion into other content domains; namely, mathematics and engineering.

\section{Mathematics Identity}

At this point in our research, recognition, interest, and the now-combined factor of performance/competence were given equal weighting in terms of their importance for identity development. However, we recognized that the impact of each factor on an individual's identity is likely not equal. So, together with colleagues studying mathematics identity, we used Structural Equation Modeling (SEM) [15] to test the theoretical structure posed for identity [16]. We found that while math recognition and interest beliefs had significant and positive direct effects on math identity, math performance/competence had an indirect effect moderated through the interest and recognition factors. Results of the SEM for physics identity were structurally identical. This work unraveled an important development in our theory: performance/competence beliefs are not sufficient for developing a physics identity - recognition and interest are necessary both for their direct influence on identity as well as for moderating the performance/competence beliefs.

Drawing from national data of college students (see Godwin \& Potvin [17] for details about the data set), we built a proxy for physics identity using only recognition and interest factors and another using recognition, interest, and performance/competence. If performance/competence only has an indirect effect through recognition and interest, then the proxy with performance/competence included should be no better at predicting physics career intentions than the proxy without it. (Note: the physics career intention variable is a fivepoint anchored scale from " 0 - Not at all likely" to " 4 Extremely likely".) In the case of the proxy with performance/competence included, the correlation of physics identity with physics career choice was $0.50(n=3679)$ with an adjusted $\mathrm{R}^{2}$ effect size of 0.25 . For the proxy with only recognition and interest factors, the correlation of physics identity with physics career choice was 0.52 $(n=4057)$ with an adjusted $\mathrm{R}^{2}$ effect size of 0.27 . Thus, the latter proxy with just recognition and interest factors has a slightly higher correlation and predicts $2 \%$ more of the variance in physics career choice, confirming that performance/competence beliefs likely have only an indirect effect on identity.

\section{Engineering Identity}

The case of engineering identity is proving to be interesting and somewhat different. In the case of physics or mathematics, students in high school and/or beginning 
college have had some direct exposure (sometimes extensive exposure) to these subjects through the courses they have taken. However, this is not the same for engineering. There are far fewer direct "engineering" experiences available for students (rare exceptions being, for example, Project Lead the Way, robotics or design competitions, etc). It has been noted for some time [18] that engineering majors may have particularly poor conceptions of their major choice before beginning college because of this.

Thus, when we set out to study students' engineering identities, we preferred to conceptualize this identity as a "pre-engineering identity", reflecting the reality that students have not had the same opportunity to develop an idea of the role of a good engineering student [19]. A structural equation model was conducted which modeled engineering identity as being influenced by both physics and mathematics identity (each of which sub-constructs showed the same internal structure for both physics and mathematics identities as described in the previous subsection) with direct, significant paths from physics and mathematics identity to engineering identity [20]. In fact, the physics identity path had a larger effect on engineering identity than mathematics identity (direct path coefficients of 0.815 and 0.537 , respectively). This model, which also includes a general science identity factor of the same structure as physics and mathematics identity, explains over $20 \%$ of the variance in the outcome of engineering career choice. Thus, these results indicate that students who ultimately choose engineering careers may be influenced through their physics and mathematics experiences (and resultant identity development in those domains), which paints a significantly different picture than in the case of physics or mathematics career choice.

\section{FUTURE WORK}

One area of particular interest for the future, which we have not yet explored, is the overlap of experiences which lead to both physics identity development and improved learning of physics content. Hazari et al. [10] identified classroom practices which are significantly related to improved physics identities, including having seen real-world connections, answering questions or making comments, etc, which suggested things that may serve such a dual purpose. However, it is not yet clear which experiences may serve to improve students' identities and their physics content knowledge. This may be an important step towards expanding our understanding of effective classroom practices, the intellectual and personal development of physics students, and improve the recruitment and retention of students in physics especially for women and other traditionally-marginalized students, who often exhibit low physics identities.

\section{ACKNOWLEDGEMENTS}

This material is based on work supported by the National Science Foundation under Grants No. 1036617, 0952460, and 0624444. Any opinions, findings, conclusions, or recommendations do not necessarily reflect the views of the National Science Foundation.

\section{REFERENCES}

1. J. Gee, Review of Research in Education 25, 99-125 (2000).

2. M.-C. Shanahan, Studies in Science Education 45, 43-64 (2009).

3. I. Esmonde, Review of Educational Research 79, 1008-1043 (2009).

4. A. Sfard, and A. Prusak, Educational Researcher 34, 14-22 (2005).

5. H. B. Carlone, and A. Johnson, Journal of Research in Science Teaching 44, 1187-1218 (2007).

6. P. R. Aschbacher, E. Li, and E. J. Roth, Journal of Research in Science Teaching 47, 564-582 (2010).

7. H. B. Carlone, Journal of Research in Science Teaching 41, 392-414 (2004).

8. A. Lichtwarck-Aschoff, P. van Geert, H. Bosma, and S. Kunnen, Developmental Review 28, 370-400 (2008).

9. R. P. DeShon, J. Z. Gillespie, et al., Journal of Applied Psychology 90, 1096 (2005).

10. Z. Hazari, G. Sonnert, P. Sadler, and M. Shanahan, Journal of Research in Science Teaching 47, 978-1003 (2010).

11. G. Potvin, C. Beattie, and K. Paige, American Association of Physics Teachers Summer Conference (2011).

12. G. Potvin, K. Paige, and C. Beattie, National Association for Research in Science Teaching Annual International Conference (2012).

13. R Core Team, $R:$ A Language and Environment for Statistical Computing, R Foundation for Statistical Computing (2013), URL http: / / www. R-project. org/.

14. A. Bandura, Social foundations of thought and action: A social cognitive theory., Prentice-Hall, Inc, 1986.

15. Y. Rosseel, Journal of Statistical Software 48, 1-36 (2012), URL http: / /www. jstat soft . org/v48/ i $02 /$.

16. J. Cribbs, Z. Hazari, P. Sadler, and G. Sonnert, Psychology of Mathematics Education - North American (PME-NA) Chapter Conference (2012).

17. A. Godwin, and G. Potvin, Chemical Engineering Education 47, 145-153 (2013).

18. E. Seymour, and N. M. Hewitt, Talking about leaving: Why undergraduates leave the sciences, Westview Press Boulder, CO, 1997.

19. A. Godwin, G. Potvin, Z. Hazari, and R. Locke, Proceedings of 2013 American Society for Engineering Education Annual Conference and Exposition (2013).

20. A. Godwin, G. Potvin, Z. Hazari, and R. Lock, Frontiers in Education Conference (2013). 